\title{
How to implement process-oriented care
}

\section{A case study on the implementation of process-oriented in-hospital stroke care}

\author{
Leti Vos · Robert J. van Oostenbrugge • \\ Martien Limburg • Godefridus G. van Merode • \\ Siebren Groothuis
}

Received: 26 March 2008 / Accepted: 7 July 2008/Published online: 20 August 2008

(c) The Author(s) 2008. This article is published with open access at Springerlink.com

\begin{abstract}
Dutch hospitals are in the midst of a transition towards process-oriented organisation to realise optimal and undisturbed care processes. Between 2004 and 2007, the University Hospital of Maastricht conducted a case study implementing process-oriented in-hospital stroke unit care. The case study consisted of four steps: (1) process analysis; (2) identification of bottlenecks; (3) setting goals for process-oriented care, and selection of coordination measures; and (4) implementation and evaluation. Implementation of process-orientation via the coordination measures chosen streamlined the process and led to a better performance of in-hospital stroke unit care; the length of stay was reduced from 12 to 7 days, and the percentage of patients who could not be admitted to the stroke unit was reduced from $31 \%$ to $2 \%$. The implementation of coordination measures is a useful means of controlling the activities of the stroke care process from a process point of view. The coordination measures used will not automatically be applicable to other care processes because of the specific nature of each care process. Nevertheless, the general principles of visual representation, bottleneck
\end{abstract}

Presented at the conference "Quality in the Spotlight", March 2008, Antwerp, Belgium.

L. Vos · G. G. van Merode $\cdot$ S. Groothuis $(\bowtie)$

School for Public Health and Primary Care (CAPHRI),

Faculty of Health, Medicine and Life Sciences,

Department of Health Organisation Policy and Economics ,

University of Maastricht, P.O. Box 616, 6200 MD Maastricht,

The Netherlands

e-mail: s.groothuis@beoz.unimaas.nl

R. J. van Oostenbrugge $\cdot$ M. Limburg

Department of Neurology, University Hospital Maastricht and Cardiovascular Research Institute Maastricht (CARIM), University Maastricht, Maastricht, The Netherlands reduction, and elimination of waste and uncertainty would be applicable in many settings.

Keywords Process-oriented organisation . Process-analysis · Process-control

\section{Introduction}

Dutch hospitals are currently in the midst of a transition towards process-oriented organisation, in order to realise optimal and undisturbed care processes. Hospitals in the Netherlands traditionally have a functional structure. Similar capacities are grouped in departments (units), for example, surgeons in the surgery department, clinical chemistry in laboratories, and so on [1]. The task differentiation and specialisation of physicians and, to a lesser degree, also nurses, is the main reason for the typical functional organisational design of hospitals [2, 3]. In a functionally organised hospital, each department strives to optimise its level of functioning, but is unable to integrate its services to best meet the needs of patients [4], i.e. one department is not able to tune its processes to those in other departments. In a process-oriented organisation, processes are mapped so that task responsibilities are described with a focus on processes. In organisation of this type, functional borders are crossed, and all members of different departments are encouraged to collaborate and achieve common goals [5].

There are two ways to implement process-oriented organisation of care: (1) implementation of coordination measures (such as care programs), or (2) clinical directorates [3,5]. In the former, horizontal processes are placed on top of the existing vertical structure, without changing the functional organisation [5]. The implementation of 
clinical directorates, on the other hand, requires changes in the hospital structure. Clinical directorates are intermediate organisational arrangements through which certain parts of larger hospitals are managed. The hospital organisation is then divided into multidisciplinary organisational units, bringing together physicians, nurses, paramedical, administrative, and clerical staff. These multidisciplinary organisational units handle a business process as comprehensively as possible and have relatively few interdependencies with other clinical directorates. With both strategies, it is necessary to overcome the functional division of labour $[3,6]$.

Until now, the implementation of process-oriented organisation of care in Dutch hospitals has been limited to the implementation of coordination measures. The implementation of directorates is hampered by, for example, (1) political and ethical obligations, which prevent hospitals from deleting services, and focussing on strategically important services with the same freedom as firms in other industries [3]; and (2) physicians' strong orientation to professional values and their commitment to developing the power and prestige of their profession, which often conflicts with managerial goals associated with processbased organisation [3].

The introduction of process-orientation in a functionalistic structure is complex. In a functional structure, the organisation of care delivery focuses traditionally only on certain elements of care rather than the whole care process. The result is a very complex system of flows and queues. There are many interactions between workplaces, and therefore many transfers occur. As a consequence, the throughput times of patient flows are unpredictable, and the quality of care is less then optimal [4]. In order to implement process-oriented care in a functional structure, management and health-care professionals must handle an additional focus: besides their focus on optimising unit performance, they also need to focus on the control of care processes across hospital units. To optimise unit performance (utilisation of resources within units), care demand and care delivery have to be adjusted. To maximise the control of care processes, waste in the patient flow over functional borders of separate units must be eliminated. Waste can be considered as activities, such as waiting and movement of staff and patients, that do not add value to a care process [7]. To maintain focus on both these aspects, management and health-care professionals have to find the appropriate trade-off between optimal unit performance (utilisation of resources within units), and the level of service provided to stroke patients (short waiting time for diagnostic tests or consultation with other medical specialties, and throughput times).
In this article, we report on a case study of the implementation of process-oriented care using coordination measures for an in-hospital stroke unit.

\section{Case study: process-oriented in-hospital stroke care}

In 2004 the University Hospital Maastricht (UHM) decided to implement process-orientation for stroke patients in order to adjust their services to the needs of these patients and to optimise the organisation of in-hospital stroke unit care. At that time the average length of hospital stay (LOS) for stroke patients was 12 days, which, according to the management of the department, surpassed the optimal LOS. According to the management of the department, the reason for this was a lack of process-orientation, resulting in poor adjustment of care delivery to the needs of the patients and inefficient use of hospital resources [8]. The importance of well organised stroke care at a specialised hospital ward (stroke unit) has been highlighted by several studies [9-12]. Admission to a stroke unit leads to improved health, functional outcomes, and survival $[9,13]$. In contrast to awareness of the qualitative components of effective stroke unit care, less attention has been paid to the operational management of stroke unit care. Little is known about effective measures that could control the whole care process from a process point of view, simultaneously optimising the efficiency of the departments involved.

In the case of in-hospital stroke care, the hospital is confronted with an acute care demand. Over $70 \%$ of stroke patients are admitted to hospital in the acute phase, resulting in uncertainty about the type and extent of care needed. This requires that the hospital has spare (reactive) capacity. Besides uncertainty over arrival times, the type of care needed by patients at the stroke unit is also complex due to the multitude of co-morbidities, which in turn requires the involvement of several medical specialties and paramedical disciplines. The uncertainty and complexity of care for stroke patients complicate the adjustment between care demand and care delivery. In the case of process-orientation this adjustment will also be dependent on the collaboration between several departments, all of which are involved in delivering care to stroke patients (neurologists, diagnostic capacities, and allied health care professionals such as occupational therapists and physiotherapists). The central idea of process-orientation is, after all, the optimisation of patient flow over the functional borders of the separate units involved. In this study, we explored the possibilities of implementing process-orientation using coordination measures. 


\section{Methods}

In order to implement process-orientation for care in an inhospital stroke unit, we used a time-series design consisting of four steps:

1. process-analysis,

2. identification of bottlenecks,

3. setting goals for process-oriented care and selection of coordination measures to realise process-oriented care,

4. implementation of coordination measures and evaluation of effects.

Each of these steps is discussed in more detail below.

\section{Step 1: Process-analysis}

In 2004 we undertook a process-analysis of the stroke care process. Our retrospective analysis was confined to patients admitted to the UHM between June and December 2003. These patients were selected retrospectively from the stroke service database of the UHM. Analysis of the existing care process comprised several steps:

(a) analysis of the UHM protocol and guidelines of the Dutch Institute for Healthcare Improvement (CBO) for stroke treatment [14],

(b) observation of the care process and structured interviews with the care providers involved,

(c) a quantitative description of the processes based on the records of stroke patients.

We collected the following demographic data on all included patients: age, sex, stroke type [based on the international statistical classification of diseases 9th revision-ICD-9-cm [15]: ischemic stroke (ICD-9-cm 434.9, 435, 436) and intracerebral haemorrhage (ICD-9-cm 431)], length of stay (time period between admission and discharge, in days), ward of admission (stroke unit or other), and discharge destination (home, nursing home, rehabilitation facility). The medical files, the hospital patient information system, and paramedical administrations were checked for the activities listed to determine whether these took place according to protocol. For each diagnostic test or therapeutic activity we determined the day of request and day of performance. We calculated the average time between request and performance. The interrelationship between the day of multidisciplinary meeting and LOS was computed per discharge destination with Spearman's correlation coefficient. We visualised the average care process in a Gantt chart-a graphical representation of the duration of tasks against the progression of these tasks [16] that aids understanding of the workflow in the stroke care process.
Step 2: Identification of bottlenecks

The performance of the in-hospital care process of stroke patients before the redesign was determined by comparing treatment protocols according to the UHM protocol and Dutch stroke guidelines [14] with actual patient treatment schedules obtained from patient records. We also examined whether all required actions were taken, and elucidated time intervals between these actions.

Step 3: Goals for process-oriented care and selection of interventions

We evaluated the identified bottlenecks with all health care professionals, including allied health workers, with the objective of improving patient flow in in-hospital stroke care in order to deliver optimal care. From an operations management point of view, optimal care can be delivered only with the right trade-off between optimal unit performance (utilisation of resources in individual units) and the service level provided to stroke patients (short waiting and throughput times).

Step 4: Implementation of redesign and evaluation of effects

In January 2006 the redesigned stroke care process to realise process-oriented care was implemented. Processanalysis and selection of interventions took a long time because many different health care professionals were involved in the process and the implementation of the process-orientation meant a shift in responsibilities for some of the professionals involved. Also, staff education was needed, which again took time.

Retrospective analysis to determine the performance after redesign was performed for patients admitted to the stroke unit of the UHM between January and April 2006. The length of this interval was limited to 4 months for practical reasons. Demographic data from all included patients were gathered. First, demographic data (sex, age, and diagnosis) were compared with independent samples by $t$-test. Next, we compared the performance of the care process for stroke patients before and after implementation of process-oriented care. Data concerning the LOS of patients admitted before and after the redesign were compared with the Mann-Whitney $U$ test.

\section{Results}

The following sections present the results of the case study according to the four steps of the time-series design. 
Table 1 Characteristics of patients included before and after implementation of process-orientation

\begin{tabular}{lll}
\hline & Before (2004) & After (2006) \\
\hline Number of patients included & 100 & 51 \\
Intracerebral haemorrhage (\%) & $15(15)$ & $7(14)$ \\
Ischemic stroke (\%) & $85(85)$ & $44(86)$ \\
Sex (number) & & \\
Men (\%) & $56(56)$ & $25(49)$ \\
Women (\%) & $44(44)$ & $26(51)$ \\
Average age (SD) in years & $74.0(9.5)$ & $73.6(12.9)$ \\
Length of hospital stay & & \\
Average (SD) in days & $12.0(6.3)$ & $7.3(5.1)$ \\
Range in days & $3-33$ & $1-30$ \\
\hline
\end{tabular}

a Total duration of hospital stay

Step 1: Process-analysis

We included 100 stroke patients. Table 1 shows the demographic data of the population studied. The average age was 74.0 years [standard deviation (SD) 9.5 years], $56 \%$ were male, $85 \%$ had an ischemic stroke, and $15 \%$ an intracerebral haemorrhage. The length of stay varied from 3 to 33 days, with the average length of stay being 12.0 days (SD 6.3 days). Due to shortage of capacity on the stroke unit, 31 patients were initially admitted to a nonspecialised ward of the UHM and subsequently transferred to the stroke unit.

The frequency of application of diagnostic examinations and consultations are shown in Table 2. The care process starts in the emergency unit. After history taking, physical examination, standard blood testing, ECG, and brain CTscan, patients are usually admitted to the stroke unit but in some cases first to another ward (all within the first $24 \mathrm{~h}$ ). The rehabilitation process, which includes mobilisation, physical therapy, occupational therapy, and speech therapy, starts in the following days, and additional examinations take place on average on day 4 , having been requested on day 2 .

The Gantt chart of the existing care process (Fig. 1) shows at least 3 days in hospital in which relatively little activity takes place. These are not clinically necessary 'watch and wait' periods. This suggests that the hospital stay could be reduced by at least these 3 days in the case of stable patients.

Required diagnostic tests-serum, cholesterol, blood testing, and chest X-ray-were not performed in 20 and $34 \%$ of cases, respectively. In the case of chest X-ray, neurologists considered the protocol to be outdated [8]. The duration of the hospital stay of patients discharged to their own home correlated positively with the number of days between day of admission and discussion in a multidisciplinary meeting (Spearman's rank correlation coefficient
$0.33, n=37, P=0.05$ ). Length of hospital stay of patients discharged to other care facilities was not affected by the number of days between admission and discussion in the multidisciplinary meeting. Patients waiting for placement in a rehabilitation facility or nursing home had an extended stay in hospital (3-8 days). The number of days without medical or diagnostic intervention (with the exception of treatments such as occupational therapy) is higher (3-9 days) for the latter patients than for patients who were discharged to home.

Step 2: Identification of bottlenecks

Analysis of existing performance helped identify four main bottlenecks for optimal organisation. The first bottleneck is a lack of bed capacity on the stroke unit. One-third of our patients could not be admitted to the stroke unit. Patients spending over 5 days in a non-specialised ward resided significantly longer in hospital (Mann-Whitney $U$ test: $Z=-2.9, P<0.05)$. A more efficient organisation of hospital stay in the stroke unit may result in treating more patients at the stroke unit, and so reduce the use of nonspecialised wards. Unnecessary delays in requesting orders for diagnostic procedures, and waiting times for diagnostic tests and consultation by allied health professionals form the second bottleneck. When a patient has been admitted, orders for all standard procedures should be written immediately by the neurologist. Yet, in reality, making these requests takes an average of 3 days. The low frequency of the multidisciplinary meeting (once a week) causes an increase in the length of stay for patients that will be discharged to their homes. Finally, there are waiting times for admission to rehabilitation and nursing facilities.

Step 3: Goals for process-oriented care and identification of coordination measures

We evaluated the four main bottlenecks with the objective of improving patient flow of in-hospital stroke care in order to deliver optimal care characterised by an appropriate trade-off between optimal unit performance (utilisation of resources in the units) and the level of service provided to stroke patients (short waiting and throughput times). In our case, the bed capacity of the stroke unit was a significant bottleneck. On the other hand, waiting times for diagnostic procedures and nursing facilities increase the LOS in hospital. In order to decrease the LOS and to eliminate waiting times as much as possible, transfer from the hospital to a nursing facility also had to be subject to improvement. The health care professionals involved set the following goals for the implementation of processorientation: (1) an average LOS in the UHM of 5 days, and (2) less than $5 \%$ of all stroke patients having to be admitted 


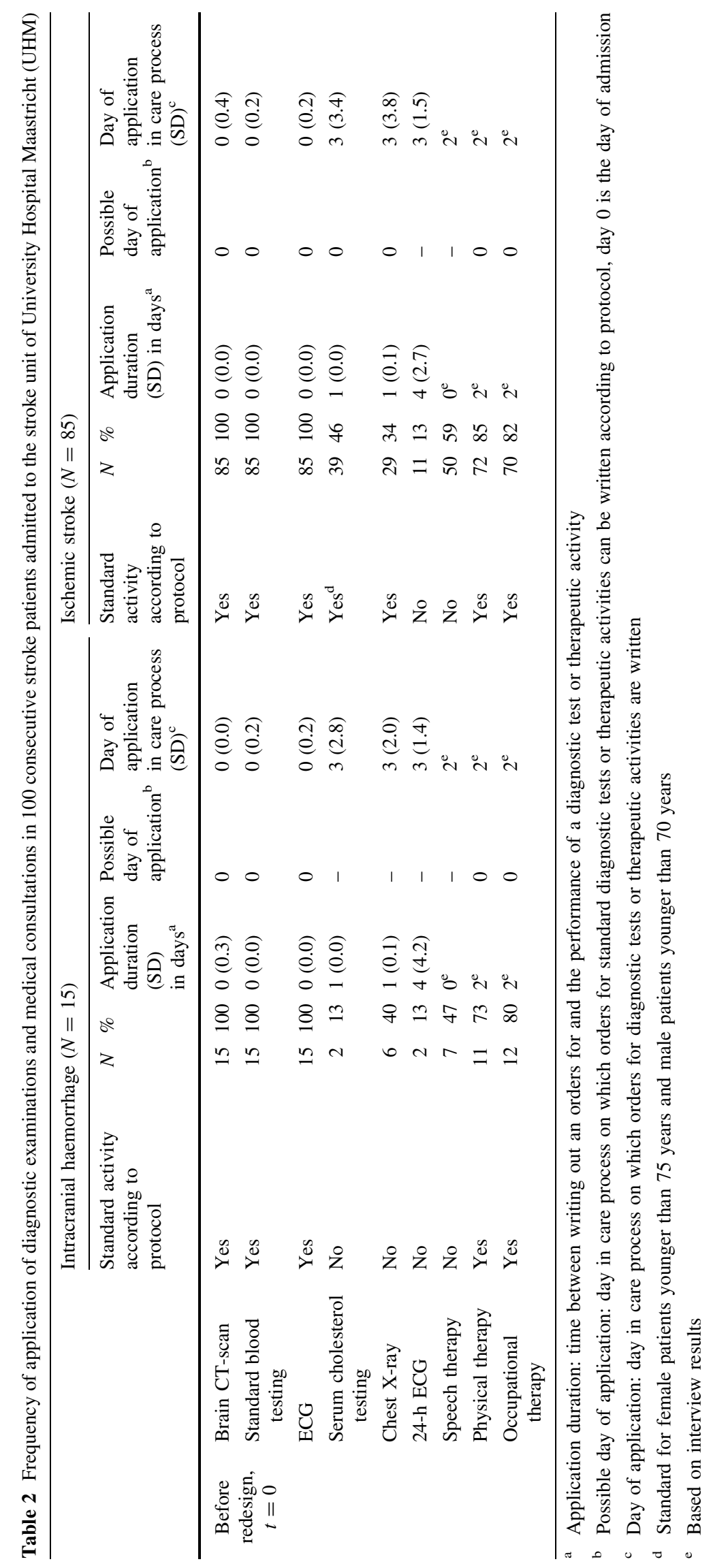




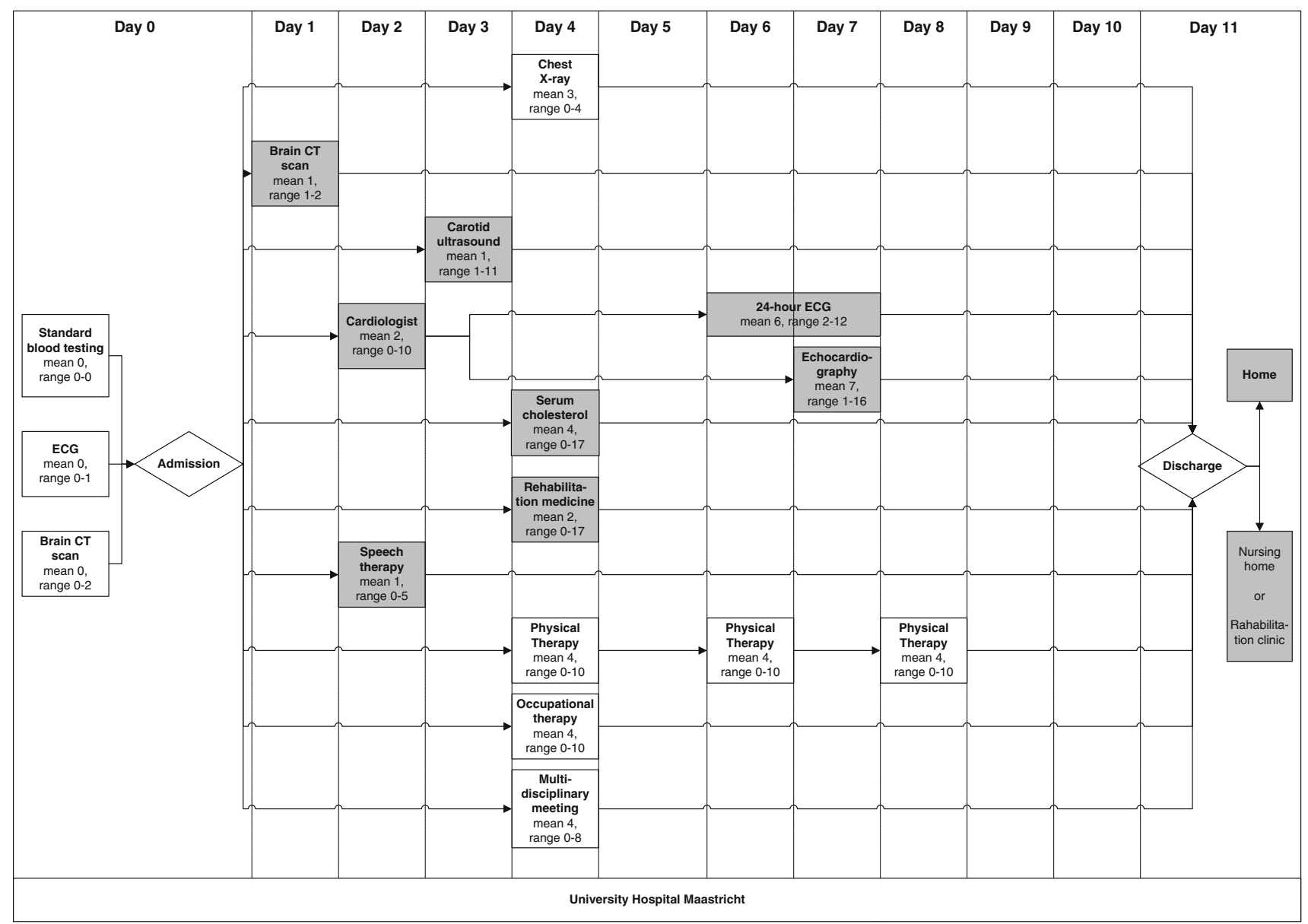

$=$ activity according to protoco

$=$ activity on request when indicated

Fig. 1 Time path of care process of 100 consecutive stroke patients admitted to the stroke unit of University Hospital Maastricht (UHM). Indicated are the average time (in days) and ranges between admission and performance of each activity

to a non-specialised ward in case of capacity shortage on the stroke unit. The following coordination measures were selected in order to achieve these goals:

- Use of an updated protocol for patients admitted to the stroke unit, with clear instructions for standard diagnostic procedures and procedures on request. The chest $\mathrm{X}$-ray was dropped as a standard activity, as this procedure is no longer considered best practice.

- Outsourcing of allied professional assessment to the rehabilitation unit of a nursing facility in order to eliminate waiting times for this assessment and treatment in the UHM, and duplication of care delivery by the UHM and the rehabilitation unit for stroke patients at a nursing facility or rehabilitation centre. As soon as patients are stabilised, they will be transferred to the nursing rehabilitation unit of a nursing facility, in order to begin assessment and rehabilitation as quickly as possible. In the UHM patients receive allied healthcare professional care on demand. In this way patients do not have to wait for placement in a nursing facility, they have direct access.

- Simplification of discharge planning. The weekly multidisciplinary meeting has been removed from the agenda in the UHM because of the delaying effect. After redesign, continuous monitoring of the patient is performed by the neurologist (in consultation with all involved disciplines) to assess the patient's condition and decide on discharge to a rehabilitation unit of a nursing home. Care delivery in the rehabilitation unit is attuned to the needs of the stroke patient. Patients whose condition allows it, will be discharged directly to their home.

The redesign of the care process is illustrated in Fig. 2.

Performance was determined by measuring the outcomes of the care process on the following formulated goals, which acted as proxies for optimal unit performance and the level of service provided to stroke patients: (1) LOS in the UHM, and (2) percentage of stroke patients 


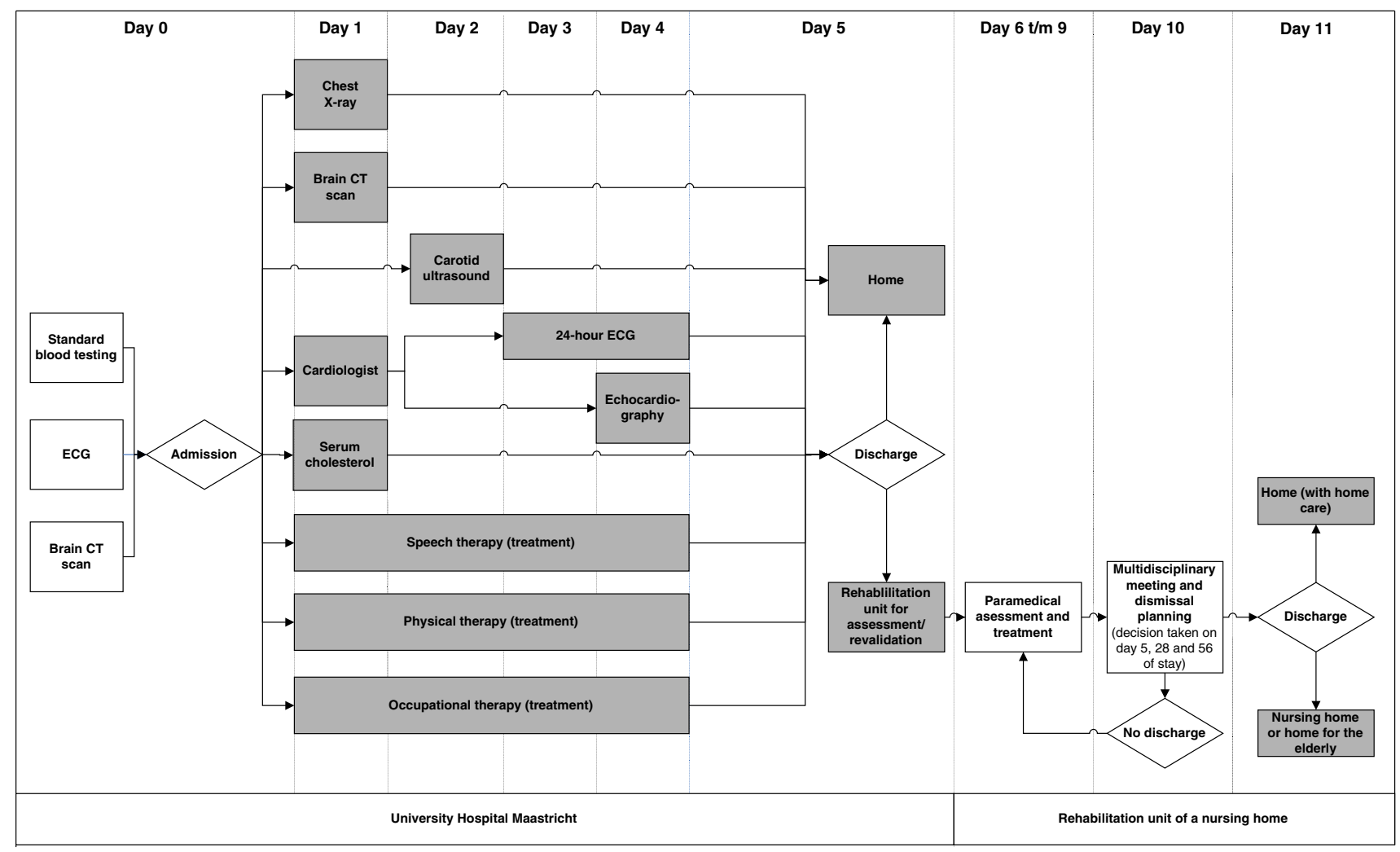

$=$ activity according to protocol

$=$ activity on request when indicated

Fig. 2 Redesign of the care process of stroke patients admitted to the UHM stroke unit

initially admitted to a non-specialised ward. These performance indicators were chosen because they oblige the professionals involved to work together as a group. If they do not cooperate, stroke care will not be optimised and waiting times will not be reduced, and consequently there will be no reduction in LOS.

Step 4: Implementation of redesign and evaluation of effects

Between January and April 2006, 51 patients were included in the study to assess the effect of the redesign. The average age was 73.6 years (SD 12.9 years), $49 \%$ of these patients were male, $86 \%$ of the patients suffered an ischemic stroke and $14 \%$ had a intracerebral hemorrhage (Table 1). There were no significant differences in demographic data of patient groups in both periods (age, $t$-test: $t=0.22$, df 149, $P>0.05$; sex, $t$-test: $t=0.58$, $d f 149$, $P>0.05$; diagnosis, $t$-test: $t=0.21, d f 149, P>0.05$ ).

Table 3 shows the results of performance indicators of the care process before and after implementation of the coordination measures. The LOS of patients admitted after the redesign varied from 1 to 30 days (average 7.3 days, $\mathrm{SD}=5.1$ days). After exclusion of three patients that were explicitly indicated for nursing home care, the average
Table 3 Performance of stroke care process in the UHM before and after implementation of process-orientation

\begin{tabular}{llll}
\hline & $\begin{array}{l}\text { Before } \\
(2004)\end{array}$ & $\begin{array}{l}\text { After } \\
(2006)\end{array}$ & $\begin{array}{l}\text { Significance } \\
\text { level }\end{array}$ \\
\hline $\begin{array}{l}\text { Average length of stay } \\
\text { (SD) in days }\end{array}$ & $12.0(6.3)$ & $7.3(5.1)$ & $P<0.01$ \\
$\begin{array}{c}\text { Percentage of patients } \\
\text { admitted to a non- } \\
\text { specialised ward }\end{array}$ & 31 & 2 & $P<0.01$ \\
\hline
\end{tabular}

length of stay was 6 days. The length of stay of patients admitted to the stroke unit was significantly shorter after implementation of the coordination measures (MannWhitney $U$ test: $Z=-5.0, P<0.01)$. Only one patient had to be admitted to another ward because of a lack of capacity on the stroke unit. This was significantly fewer compared to the number of patients admitted to nonspecialised ward before the redesign ( $t$-test: $t=8.0$, degrees of freedom $149, P<0.01$ ).

\section{Discussion}

In this study we explored the possibilities of implementing process-orientation for care in an in-hospital stroke unit 
using coordination measures. In process-oriented care, the performance of the whole care process is reviewed. To maximise performance of a care process from the point of view of process-oriented care, waste (defined as unnecessary steps in the care process) had to be eliminated [4, 7]. Besides optimising the level of service provided (short waiting and throughput times), we also needed to take the unit performance into account, since process-oriented care was to be implemented in a functionally organised hospital. In this case study, implementation of coordination measures led to better performance of in-hospital stroke unit care in terms of LOS (throughput time) and the number of patients that could be admitted to the stroke unit. Although the chosen process performance indicators (LOS, number of patients admitted to non-specialised wards) are perhaps not the best proxies for process-orientation, we conclude that the coordination measures applied here were useful in coordinating the activities of the stroke care unit from a process point of view for several reasons.

Firstly, standardisation of the in-hospital stroke care process facilitated the decision-making process by making clear which medical and diagnostic procedures were required by each patient and by whom these had to be performed in the care process. This led to better control of the in-hospital stroke care process in time. As a consequence, less flexibility in terms of spare capacity was needed, thus optimising unit performance.

Secondly, the transfer of allied professional assessment and treatment to the rehabilitation unit of a nursing facility reduced waste caused by duplication of work and unnecessary waiting times for allied professional assessment and treatment in the UHM. Besides the reduction of waste, it simplified the planning process and made clear which aspects of care were the responsibility of the specialist services.

In addition, the fact that the number of patients that could be admitted directly to the stroke unit increased after implementation reduced the complexity of planning of the care process and increased the quality of care, since admission to a stroke unit leads to improved health, functional outcomes and survival [9]. The reduction in the number of patients admitted to non-specialised wards also eliminated waste from the process because professionals no longer had to walk to the other side of the hospital to care for their patients.

We showed that application of the described coordination measures contributed to both the adjustment of care demand and care delivery, as streamlining of the process reduced uncertainty and complexity. Uncertainty and, as a consequence, the need for flexibility are reduced by making the process more transparent. Complexity is reduced by standardisation and reducing waste. Care activities are now delivered in a more integrated manner.
Furthermore, it would appear that all involved professionals now see their care delivery as part of a care process. This means that they have shifted their focus from optimisation of performance of their unit to improving process performance overall.

Although this study was quite time-consuming, all steps taken were considered essential. Visualising the care process in Gantt charts creates a sense of urgency among the involved professionals to streamline processes because they can actually see what is happening with the patient during their hospital stay.

Limitations of our study include the fact that the process-analysis and evaluation of effects did not take place at the same time of year. Seasonal factors could have influenced our results. Also, the fact that the time span between process-analysis and evaluation of effects was quite long for organisational reasons could mean that changes in hospital personnel or other changes in hospital practice could have taken place and influenced our results. However, we do not consider that these limitations biased our results as we described the process in both periods in detail and were not aware of any changes in the hospital process other than those initiated by our study.

\section{Conclusion}

The present case study demonstrates that process-orientation using coordination measures can be implemented for in-hospital stroke unit care, and can improve performance by reducing the uncertainty and complexity of this type of care. The method used to decide which coordination measures are needed could be applied to other patient groups. The coordination measures that the UHM has taken to optimise in-hospital stroke care will not be automatically applicable to other care processes because of the specific components of each care process. However, the general principles of visual representation, bottleneck reduction, and elimination of waste and uncertainty would be very useful in many settings.

Open Access This article is distributed under the terms of the Creative Commons Attribution Noncommercial License which permits any noncommercial use, distribution, and reproduction in any medium, provided the original author(s) and source are credited.

\section{References}

1. Van Merode GG (2004) A prelude of 2004 Antwerp quality conference: targets and target values-integrating quality management and costing. Accred Qual Assur 9:168-171

2. Braithwaite J (1993) Strategic management and organisational structure: transformational at work in hospitals. Aust Health Rev 16:383-404 
3. Vera A, Kuntz L (2007) Process-based organization design and hospital efficiency. Health Care Manage Rev 32:55-65

4. Van Merode F, Molema H, Goldschmidt H (2004) GUM and six sigma approaches positioned as deterministic tools in quality target engineering. Accred Qual Assur 10:32-36. doi:10.1007/ s00769-004-0876-0

5. Gemmel P, Vandaele D, Tambeur W (2007) Hospital process orientation (HPO): the development of a measurement tool. Gent: Ghent University, Belgium. http://www.feb.ugent.be/fac/ research/WP/Papers/wp_07_480.pdf. Accessed 4 February 2008

6. Vanhaverbeke W, Torremans H (1999) Organisational structure in process-based organisations. Knowledge Process Manag 6:41-52. doi:10.1002/(SICI)1099-1441(199903)6:1 $\leq 41::$ AID$\mathrm{KPM} 47 \geq 3.0 . \mathrm{CO} ; 2-4$

7. Liker JK (2004) The Toyota way: 14 management principles from the world's greatest manufacturer. McGraw-Hill, New York

8. Limburg M, Vos L, van Oostenbrugge R, van Merode GG, Groothuis S (2005) Causes of inefficient stroke unit -bed usepossibilities for freeing up capacity. Cerebrovasc Dis 19:114-115

9. Stroke Unit Trialists' Collaboration (2001) Organised inpatient (stroke unit) care for stroke. The Cochrane Database of Systematic Reviews, Issue 3

10. Kjellström T, Norrving B, Helsingborg Declaration AS (2007) 2006 on European stroke strategies. Cerebrovasc Dis 23:229241. doi:10.1159/000097646
11. Langhorne P, Pollock A (2002) What are the components of effective stroke unit care? Age Ageing 31:365-371. doi: 10.1093/ageing/31.5.365

12. Leys D, Ringelstein B, Kaste M, Hack W (2007) The main components of stroke unit care: result of a European expert survey. Cerebrovasc Dis 2007:344-352

13. Brainin M, Olsen TS, Chamorro A, Diener HC, Ferro J, Hennerici MG et al (2004) Organisation of stroke care: education, referral, emergency management and imaging, stroke units and rehabilitation. Cerebrovasc Dis 17(suppl. 2):1-14. doi:10.1159/ 000074816

14. Limburg M, Tuut MK (2000) Stroke guideline, dutch institute for health care CBO (in Dutch). Ned Tijdschr Geneeskd 144:10581062

15. Manual of the international statistical classification of diseases, injuries, and causes of death: based on the recommendations of the ninth revision conference, 1975, and adopted by the 29th World Health Assembly. World Health Organization, Geneva (1977-1978)

16. Ozcan YA (2005) Quantitative methods in health care management: techniques and applications. Jossey-Bass, San Francisco 